\title{
Graded aerobic treadmill testing in pediatric sports-related concussion: safety, clinical use, and patient outcomes
}

\author{
Dean Cordingley, MSc, ${ }^{1,2}$ Richard Girardin, CAT(c), ${ }^{1,2}$ Karen Reimer, BMR, PT, MSc, $, 1,2,7$ \\ Lesley Ritchie, PhD, ${ }^{1-3}$ Jeff Leiter, PhD, ${ }^{1,2,4}$ Kelly Russell, PhD, ${ }^{2,5,8}$ and Michael J. Ellis, MD 1,2,4-6,8 \\ ${ }^{1}$ Pan Am Concussion Program; ${ }^{2}$ Canada North Concussion Network; Departments of ${ }^{3} \mathrm{Clinical}$ Health Psychology, ${ }^{4}$ Surgery, \\ ${ }^{5}$ Pediatrics and Child Health, ${ }^{6}$ Section of Neurosurgery, and ${ }^{7}$ Section of Physical Therapy, College of Rehabilitation Sciences, \\ University of Manitoba; and ${ }^{8}$ Children's Hospital Research Institute of Manitoba, Winnipeg, Manitoba, Canada
}

OBJECTIVE The objectives of this study were 2-fold: 1) to evaluate the safety, tolerability, and clinical use of graded aerobic treadmill testing in pediatric patients with sports-related concussion (SRC), and 2) to evaluate the clinical outcomes of treatment with a submaximal aerobic exercise program in patients with physiological post-concussion disorder (PCD).

METHODS The authors conducted a retrospective chart review of pediatric patients (age $<20$ years) with SRC who were referred to a multidisciplinary pediatric concussion program and underwent graded aerobic treadmill testing between October 9, 2014, and February 11, 2016. Clinical assessments were carried out by a single neurosurgeon and included clinical history taking, physical examination, and recording specific patient-reported concussion-related symptoms using the Post-Concussion Symptom Scale (PCSS). Graded aerobic treadmill testing using a modified Balke protocol for incremental increases in intensity was used as a diagnostic tool to assess physiological recovery, classify post-concussion syndrome (PCS) subtype, and reassess patients following treatment. Patients with a symptom-limited threshold on treadmill testing (physiological PCD) were treated with an individually tailored submaximal exercise prescription and multidisciplinary targeted therapies.

RESULTS One hundred six patients (mean age 15.1 years, range 11-19 years) with SRC underwent a total of 141 treadmill tests. There were no serious complications related to treadmill testing in this study. Overall, 138 (97.9\%) of 141 tests were well tolerated and contributed valuable clinical information. Treadmill testing confirmed physiological recovery in 63 $(96.9 \%)$ of 65 patients tested, allowing successful return to play in $61(93.8 \%)$. Treadmill testing was used to diagnose physiological PCD in 58 patients and cervicogenic PCD in 1 patient. Of the 41 patients with physiological PCD who had complete follow-up and were treated with tailored submaximal exercise prescription, $37(90.2 \%)$ were classified as clinically improved and $33(80.5 \%)$ successfully returned to sporting activities. Patients who did not respond or experienced an incomplete response to submaximal aerobic exercise treatment included 7 patients with migraine headaches and 1 patient with a postinjury psychiatric disorder.

CONCLUSIONS Graded aerobic treadmill testing is a safe, tolerable, and clinically valuable tool that can assist in the evaluation and management of pediatric SRC. Future research is needed to confirm the clinical value of this tool in return-to-play decision making. Studies are also needed to understand the pathophysiology of physiological PCD and the effects of targeted treatment.

http://thejns.org/doi/abs/10.3171/2016.5.PEDS16139

KEY WORDS concussion; trauma; post-concussion syndrome; physiological post-concussion disorder; pediatric; graded aerobic treadmill testing; multidisciplinary

ABBREVIATIONS ADHD = attention deficit/hyperactivity disorder; $\mathrm{BP}=$ blood pressure; HR = heart rate; ICD-10 = International Classification of Diseases, 10 th Revision; ICHD-3 beta = International Classification of Headache Disorders, 3rd edition (beta version); ImPACT = Immediate Post-Concussion Assessment and Cognitive Testing; IQR = interquartile range; PARmed-X = Physical Activity Readiness Medical Examination; PCD = post-concussion disorder; $P C S=$ post-concussion syndrome; PCSS = PostConcussion Symptom Scale; RPE = rating of perceived exertion; RR = respiratory rate; SRC = sports-related concussion.

SUBMITTED March 9, 2016. ACCEPTED May 18, 2016.

INCLUDE WHEN CITING Published online September 13, 2016; DOI: 10.3171/2016.5.PEDS16139. 
$\mathrm{C}$ LINICAL studies suggest that $21 \%-73 \%$ of children and adolescents who sustain a concussion will experience persistent symptoms at 1 month after injury or develop post-concussion syndrome (PCS). ${ }^{2,9,11,12,21,40}$ Although laboratory model studies suggest that acute concussion is characterized by a global metabolic energy crisis,$^{20}$ there are few clinical diagnostic tools that provide a view into the pathophysiology of $\mathrm{PCS}^{13}$ and few evidencebased treatment options for patients with this condition. ${ }^{6,7}$

Over the past 5-10 years, graded aerobic treadmill testing has emerged as a safe and reliable clinical tool that can assist in the evaluation and treatment of patients with acute concussion and PCS. Using a standardized incremental treadmill-based exercise protocol, this testing method can be used to assess physiological concussion recovery, distinguish between post-concussion disorders (PCDs)-physiological PCD, vestibulo-ocular PCD, and cervicogenic PCD - and direct patients toward treatment options that target the pathophysiological processes underlying persistent symptoms. ${ }^{14,25,28,29}$ Patients who achieve a symptom-limited threshold during treadmill testing are diagnosed with physiological PCD, a condition associated with alterations in regional cerebral blood flow and cerebrovascular responsiveness. ${ }^{8,35}$ Submaximal aerobic exercise has been shown to be a safe and effective treatment option resulting in symptomatic recovery and return to sport activities in the vast majority of adult patients with PCS. ${ }^{3,26}$ Despite these advances, there have been no studies that have examined the safety, tolerability, and clinical use of graded aerobic treadmill testing in an exclusive cohort of pediatric concussion patients. Furthermore, there have been no studies that have examined the clinical outcomes of pediatric patients with physiological PCD treated with submaximal aerobic exercise and targeted multidisciplinary rehabilitation strategies.

Accordingly, the primary objective of this retrospective study was to evaluate the safety, tolerability, and clinical use of graded aerobic treadmill testing in pediatric patients with sports-related concussion (SRC) evaluated and treated at a multidisciplinary pediatric concussion program. The secondary objective was to evaluate the clinical outcomes in patients with physiological PCD treated with submaximal aerobic exercise prescription. The contribution of graded aerobic treadmill testing to our evolving institutional multidisciplinary approach to pediatric acute SRC and PCS is also discussed.

\section{Methods}

\section{Study Design and Inclusion Criteria}

A retrospective chart review was performed for all pediatric patients evaluated at the Pan Am Concussion Program at the Pan Am Clinic, Winnipeg, Manitoba, Canada, between October 9, 2014, and February 11, 2016, who underwent graded aerobic treadmill testing. To qualify for inclusion in the study, patients had to be 19 years or younger at the time of testing and have a diagnosis of acute SRC or PCS secondary to an SRC. Patients with non-sportsrelated injuries, those with moderate or severe traumatic brain injuries, and those with traumatic abnormalities detected on clinical neuroimaging studies were excluded.
This study was approved by the institutional ethics review board at the University of Manitoba.

\section{Definitions}

The diagnosis of concussion was made according to the International Consensus on Concussion in Sport definition as an injury caused by transmission of biomechanical forces to the brain leading to clinical symptoms affecting multiple domains of physical, cognitive, sleep, and neurobehavioral functioning. ${ }^{34}$ Post-concussion syndrome (PCS) was diagnosed in patients who reported 3 or more symptoms identified by the International Classification of Diseases, 10th Revision (ICD-10) ${ }^{4}$ and remained symptomatic for 1 month or longer postinjury. Patients with SRC were diagnosed with coexisting vestibulo-ocular dysfunction if they reported more than 1 subjective vestibular or oculomotor complaint (dizziness, blurred vision, etc.) and demonstrated more than 1 abnormal finding on physical examination (e.g., abnormal smooth pursuits, saccades, near point of convergence, abnormal vestibuloocular reflex) as previously described. ${ }^{12}$

\section{Clinical Assessments}

At initial medical consultation, patients (with assistance of their parents in some cases) completed a standardized data collection form that included demographic data, medical history, past concussion history, and family history, as well as the Post-Concussion Symptom Scale (PCSS), a self-reported symptom inventory that includes 22 symptoms rated on a 7-point (0-6) Likert scale with a maximum score of 132. A single neurosurgeon (M.J.E.) obtained a clinical history and performed a complete physical examination in all cases. The physical examination included evaluation of cranial nerve, motor, sensory, cerebellar, gait, balance, cognitive, and cervical spine function. A standardized, focused vestibulo-ocular clinical examination using previously described techniques ${ }^{12}$ was also performed on all patients. In general, patients with acute concussion were treated with conservative management for the first 2-4 weeks with appropriate levels of physical and cognitive rest as well as return-to-learn and return-to-play guidance. Patients who developed persistent symptoms lasting longer than 2-4 weeks and those referred with a diagnosis of PCS were often managed using a multidisciplinary approach.

In selected cases, confirmation of concussion recovery was supplemented by the use of a hybrid neuropsychological testing approach (computerized neurocognitive tools and/or pencil-and-paper instruments) interpreted by a registered clinical neuropsychologist. All formal neuropsychological testing, graded aerobic treadmill testing, and referrals to multidisciplinary specialists were initiated based on the clinical discretion of the treating neurosurgeon and availability of the clinical neuropsychologist and not according to predetermined research criteria.

\section{Graded Aerobic Exercise Treadmill Testing}

Patients were referred for graded aerobic treadmill testing for the following clinical indications: 1) assessment of exercise tolerance and classification of PCS subtype 
(physiological PCD, vestibulo-ocular PCD, or cervicogenic PCD); 2) assessment of physiological recovery; and 3 ) reassessment following prior graded aerobic treadmill testing and submaximal exercise prescription.

Prior to graded aerobic treadmill testing, all patients underwent medical screening performed by the neurosurgeon, who used the Physical Activity Readiness Medical Examination (PARmed-X). This examination includes assessment of resting blood pressure (BP) and heart rate (HR), as well as screening for medical conditions (cardiovascular, respiratory, neurological, etc.) that could present absolute or relative contraindications to exercise testing. All participants were fitted with a Zephyr Echo bioharness (Zephyr Technology), which was affixed to the patient's chest and recorded real-time HR, respiratory rate (RR), and HR variability throughout the test. Informed consent was obtained from patients and their parents prior to testing. Potential risks that were disclosed to the patient and their parents included the risk of temporary worsening or exacerbation of concussion symptoms, the risk of falling off the treadmill and sustaining injury, the risk of shortness of breath and muscle soreness related to exercise, and the risk of skin irritation related to the physiological bioharness monitor.

Following medical clearance and informed consent, all patients underwent Buffalo Concussion Treadmill Testing in accordance with previously described protocols. ${ }^{23,24}$ Graded aerobic treadmill testing was undertaken by an experienced exercise physiologist and athletic therapist under the supervision of the neurosurgeon. Using a modified version of the standardized treadmill-based Balke protocol, patients performed an incremental series of exercises to the point of symptom onset or exacerbation or until maximal exhaustion was achieved (defined as a rating of perceived exertion [RPE] of $18-20$ on the Borg scale). ${ }^{5}$ Specifically, patients began walking on the treadmill at a speed of $3.2 \mathrm{mph}$ and $0 \%$ grade. The grade was increased by $1 \%$ per minute for the first 15 minutes, after which time the speed was increased $0.2 \mathrm{mph}$ per minute. Patients were asked to rate their symptoms and RPE every minute. Each patient underwent continuous HR monitoring throughout the test. Blood pressure was assessed immediately following test termination and again following a 5-minute cooldown period.

Based on the results of graded aerobic treadmill testing and physical examination findings, patients were classified into 4 clinical categories: 1) physiologically recovered (reached maximal exertion without onset of concussion symptoms); 2) physiological PCD (developed a symptomlimited threshold during testing); 3) vestibulo-ocular PCD (did not reach a symptom-limited threshold during testing but met the clinical criteria for vestibulo-ocular dysfunction): ${ }^{12}$ and 4 ) cervicogenic PCD (did not reach a symptomlimited threshold during testing but demonstrated evidence of coexisting cervical spine soft tissue injury on clinical history and physical examination-i.e., neck tenderness, reduced range of motion, or cervical musculature spasm). ${ }^{14}$

Patients who met the clinical criteria for physiological PCD were prescribed a tailored submaximal exercise program to be followed 5 days a week. The program consisted of a 5-minute warm-up, 20 minutes of aerobic exercise at
$80 \%$ of the maximum HR achieved during treadmill testing (or equivalent RPE), and a 5-minute cooldown. Patients were seen in follow-up every $2-4$ weeks, at which time repeat treadmill testing, transition into a sport-specific return-to-play program, or initiation of other multidisciplinary intervention was considered. Repeat treadmill testing was considered by the neurosurgeon on an individual basis for patients who demonstrated clinical improvement but reported persistent concussion symptoms following initial submaximal aerobic exercise prescription. Repeat treadmill testing allowed the treatment team to reassess exercise tolerance in these patients and safely increase the HR and exercise intensity targets of the submaximal exercise program. In cases in which concussion symptoms resolved after submaximal exercise prescription, selected patients underwent graded aerobic treadmill testing to confirm physiological recovery or were transitioned into sport-specific return-to-play programs at the discretion of the treating neurosurgeon. Patient compliance with the submaximal exercise prescription was not formally monitored with logbooks or digital watches but only through clinical follow-up with the treatment team.

\section{Graded Aerobic Treadmill Testing and Patient Outcomes}

Complications related to graded aerobic treadmill testing were recorded. Patients who were treated with submaximal aerobic exercise prescription were classified as "clinically improved" if they reported a subjective improvement in concussion symptoms and/or showed a reduction in PCSS scores during clinical follow-up visits after the initiation of an exercise program. Patients were classified as "not clinically improved" if they did not report a subjective improvement in concussion symptoms and/or show a reduction in PCSS scores during follow-up after the initiation of an exercise program. Patients were classified as having an "incomplete response" if they experienced initial clinical improvement with submaximal exercise prescription but did not achieve full recovery. In general, patients were classified as fully recovered when they were asymptomatic at rest according to the clinical interview and PCSS, were participating in full-time school activities without symptoms, had normal findings on neurological examination, and did not meet the clinical criteria for vestibulo-ocular dysfunction. In selected patients treated with submaximal exercise prescription, repeat graded aerobic treadmill testing and/or neuropsychological testing was used to confirm physiological recovery. All computerized neurocognitive and formal neuropsychological testing was performed on the day of, but prior to, treadmill testing or more than 1 day afterward. For patients who were returning to in-season sports, participation was managed according to the International Consensus on Concussion in Sport graduated return-toplay protocol. ${ }^{34}$ All patients were advised to return to the concussion program if they developed any recurrent concussion symptoms following medical clearance to return to sports.

\section{Statistical Analysis}

The distributions of baseline characteristics for SRC 
patients who underwent graded aerobic treadmill testing were summarized using proportions for dichotomous/ polytomous characteristics and means with standard deviations for continuous characteristics. If a continuous variable was not normally distributed, it was summarized using the median and interquartile range (IQR). Therapeutic interventions and outcomes were tabulated.

\section{Results \\ Participants}

One hundred six SRC patients who underwent graded aerobic treadmill testing at our multidisciplinary pediatric concussion program were included in the study. The mean age of the study patients was 15.1 years (SD 1.5 years, range 11-19 years) and $46(43.4 \%)$ of the patients were male. The patients' medical histories were notable for previous concussion in 61 cases $(57.6 \%)$, migraine or nonspecific headaches in 23 cases $(21.7 \%)$, attention deficit/ hyperactivity disorder (ADHD) in 6 cases $(5.7 \%)$, preinjury mood disorder in 3 cases (2.8\%), nonspecific learning disorder in 2 cases $(1.9 \%)$, and asthma in 5 cases (4.7\%). Hockey (played by 23 patients [50.0\%]) and football (played by 12 [26.1\%]) were the most commonly played sports at the time of injury for male patients, and hockey (played by 18 patients [30.0\%]) and soccer (played by 7 [11.7\%]) were the most common sports for female patients. Loss of consciousness was reported in 13 cases (12.3\%), posttraumatic amnesia was reported in 35 (33.0\%), and delayed symptom onset was reported in 44 (41.5\%). The median PCSS score at initial consultation for this cohort was 25 (IQR 13.75-48). Coexisting neurological disorders diagnosed in patients who underwent treadmill testing included traumatic cranial neuropathy (optic in 1 case and trochlear in 1 case). Two patients were taking medications to target posttraumatic headaches at the time of initial treadmill testing.

\section{Safety and Tolerability of Graded Aerobic Treadmill Testing}

In this study, 106 pediatric patients with SRC completed a total of 141 graded aerobic treadmill tests (mean number of tests per patient 1.3 , SD 0.6 , range 1-4).

There were no serious complications related to treadmill testing in this study. Overall, 138 (97.9\%) of 141 tests were completely tolerated; 3 tests were terminated early due to transient and self-limiting lower-extremity soreness related to previous orthopedic injuries. The mean duration of all treadmill tests was 18.1 minutes (SD 6.7 minutes, range 4-34 minutes). None of the patients experienced persistent elevations in any of the physiological parameters monitored, including HR, BP, and RR. There were no adverse cardiorespiratory events (e.g., presyncope or asthma attack) during or after treadmill testing among patients with known cardiac or respiratory conditions. No adverse events were observed during or after treadmill testing in the 2 patients with coexisting cranial neuropathies. No patient required delayed medical attention for acute exacerbation of concussion symptoms following treadmill testing.

\section{Clinical Use of Graded Aerobic Treadmill Testing and Patient Outcomes}

Clinical indications for the 141 graded aerobic treadmill tests completed in this cohort included: assessment of physiological recovery (65 tests [46.1\%]); assessment of exercise tolerance and classification of PCS subtype (61 tests [43.3\%]); and reassessment after submaximal exercise prescription (15 tests [10.6\%]). Overall, 138 (97.9\%) of the 141 treadmill tests contributed clinically useful information that directly affected patient management. Three tests ( 2 completed for PCS classification and 1 for assessment of physiological recovery) were terminated early due to transient and self-limiting lower-extremity soreness, leading to indeterminate results that could not be used to inform clinical decision making in these cases.

In this study, 65 graded aerobic treadmill tests were completed to assess physiological recovery, including 45 in patients who underwent initial graded aerobic treadmill testing to confirm physiological recovery and 20 in patients with physiological PCD who underwent repeat treadmill testing to confirm recovery following treatment with submaximal exercise. The median days from injury to clinical consultation among the 45 patients who underwent initial treadmill testing for confirmation of physiological recovery was 8 days (IQR 5-22 days). The median time from injury to treadmill testing among these patients was 35 days (IQR 18-78 days).

Overall, treadmill testing confirmed physiological recovery in 63 (96.9\%) of 65 patients, and 2 patients (3.1\%) were deemed not physiologically recovered. In this study, a subgroup of 23 patients with SRC underwent graded aerobic treadmill testing and neuropsychological testing to confirm physiological and neurocognitive recovery. Twelve patients underwent treadmill testing and Immediate Post-Concussion Assessment and Cognitive Testing (ImPACT) testing for medical clearance. Ten patients who were deemed physiologically recovered by treadmill testing demonstrated ImPACT scores within normal limits and were medically cleared for return to sports. A patient deemed physiologically recovered by treadmill testing demonstrated 1 ImPACT subtest score in the borderline range and was medically cleared following repeat ImPACT testing 1 week later. Finally, 1 patient deemed not physiologically recovered demonstrated 2 ImPACT subtest scores in the less-than-5th-percentile range and was not cleared for return to sports. Eleven patients underwent treadmill testing and formal neuropsychological testing using pencil-and-paper tests. Nine patients deemed physiologically recovered by treadmill testing demonstrated results within normal limits, allowing medical clearance for return to sports. Two patients who were considered physiologically recovered on the basis of treadmill testing demonstrated multidomain cognitive impairments consistent with concussion on formal neuropsychological testing and were not cleared for a return to sports. Among the 61 patients who were medically cleared to return to sports, $100 \%$ returned to sports without any complication and none returned to the concussion program for evaluation or treatment of recurrent symptoms during the study period.

Among the 61 patients who underwent graded aerobic treadmill testing to classify PCS subtypes, 58 (95.1\%) 


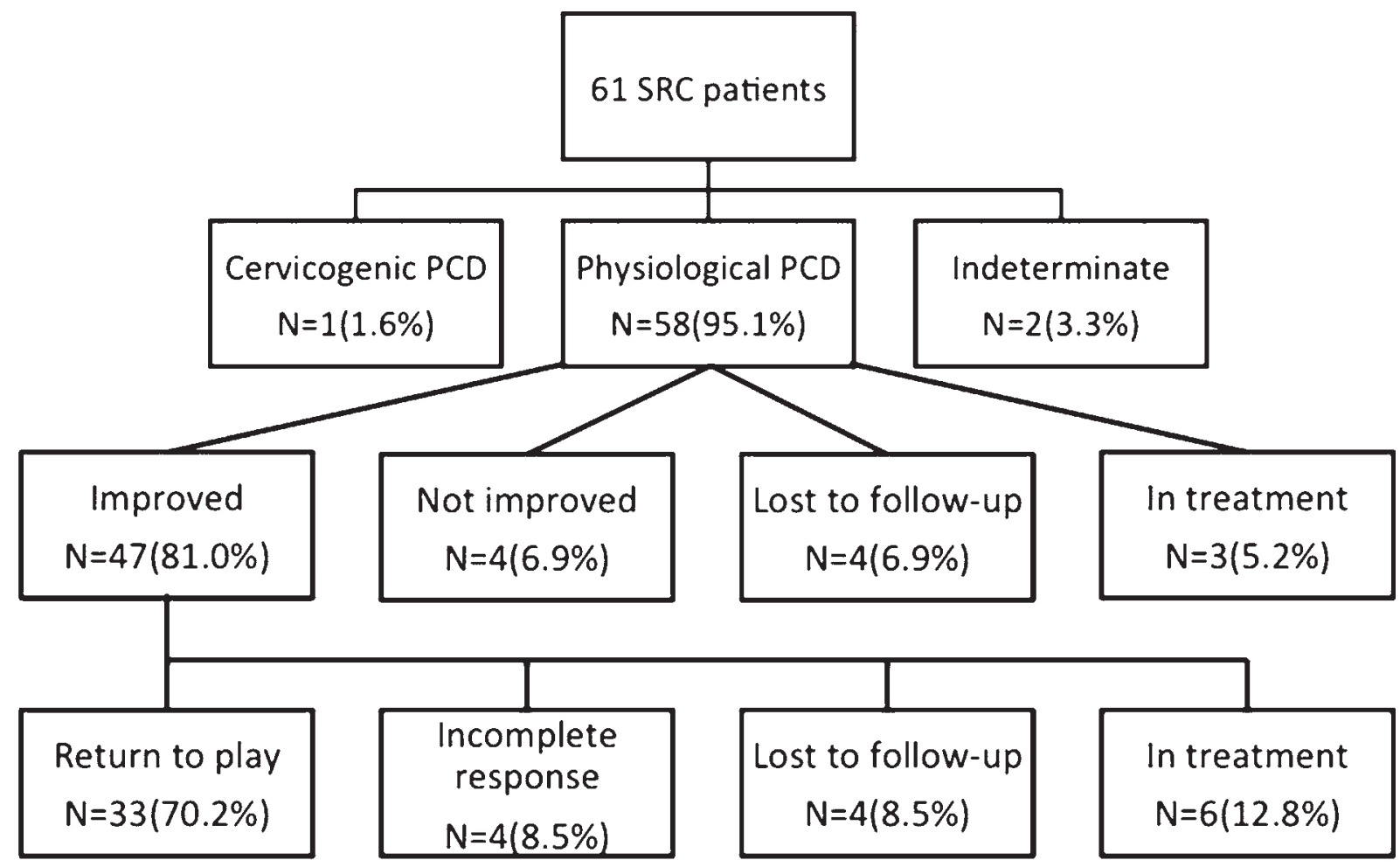

FIG. 1. Clinical outcomes in 61 cases involving pediatric patients with sports-related concussion (SRC) who underwent graded aerobic treadmill testing for assessment of exercise tolerance and post-concussion syndrome (PCS) subtype classification. PCD = post-concussion disorder.

were diagnosed with physiological PCD and $1(1.6 \%)$ was diagnosed with cervicogenic PCD (Fig. 1). The median time from injury to clinical consultation among the 61 patients who underwent treadmill testing for classification of PCS subtype was 28 days (IQR 10-62 days). Of the 58 patients who were diagnosed with physiological PCD, all were prescribed tailored submaximal aerobic exercise programs. Of those patients with physiological PCD, 24 (41.4\%) underwent submaximal aerobic exercise prescription alone, $20(34.5 \%)$ underwent submaximal exercise therapy and concurrent targeted vestibular rehabilitation for coexisting vestibulo-ocular dysfunction, 6 (10.3\%) underwent submaximal exercise therapy and concurrent cervical spine physiotherapy for coexisting cervicogenic symptoms, and 8 (13.8\%) underwent submaximal exercise therapy and concurrent vestibular rehabilitation and cervical spine physiotherapy. Following initial treadmill testing and submaximal exercise prescription, $47(81.0 \%)$ of the 58 patients with physiological PCD were classified as clinically improved, 4 (6.9\%) were classified as clinically not improved, 4 (6.9\%) did not return for follow-up after initial treadmill testing, and $3(5.2 \%)$ remained in treatment at the end of the study period. Among the 47 patients who were clinically improved, $33(70.2 \%)$ met the clinical criteria for complete recovery and returned to sports, 4 (8.5\%) experienced an incomplete response, $4(8.5 \%)$ were lost to follow-up, and $6(12.8 \%)$ remained in treatment at the end of the study period (Fig. 1). Clinical outcomes for physiological PCD patients with complete follow-up who were treated with submaximal exercise therapy alone or with submaximal exercise therapy and targeted multidis- ciplinary interventions are summarized in Fig. 2. Among the 41 patients with physiological PCD who were treated with tailored submaximal exercise and had complete follow-up, $37(90.2 \%)$ were classified as clinically improved and $33(80.5 \%)$ successfully returned to sporting activities. The median duration of submaximal aerobic exercise prescription among patients with physiological PCD who successfully returned to play was 50 days (IQR 32-123 days). Among the 8 patients with physiological PCD who did not respond to the initial submaximal exercise prescription or experienced an incomplete response after initial clinical improvement, 7 were referred to a headache neurologist for pharmacological management of headaches that met the International Classification of Headache Disorders, 3rd edition (beta version) (ICHD-3 beta) criteria for migraine, including 1 patient with new-onset migraine headaches and 6 patients with a preinjury history of migraine headaches or nonspecific headaches who developed worsening headaches. The remaining patient who did not respond to submaximal exercise prescription had prominent emotional symptoms and had undergone recent referral to an adolescent psychiatrist resulting in the diagnosis of major depressive disorder, generalized anxiety disorder, and ADHD. This patient was treated with a combination of pharmacological and behavioral interventions. Of the 2 patients who underwent treadmill testing while being treated with headache medications, one experienced an incomplete response to submaximal exercise therapy and continued the medication regimen and the other was successfully weaned from headache medication and returned to play following treadmill testing-confirmed physiological recovery. 


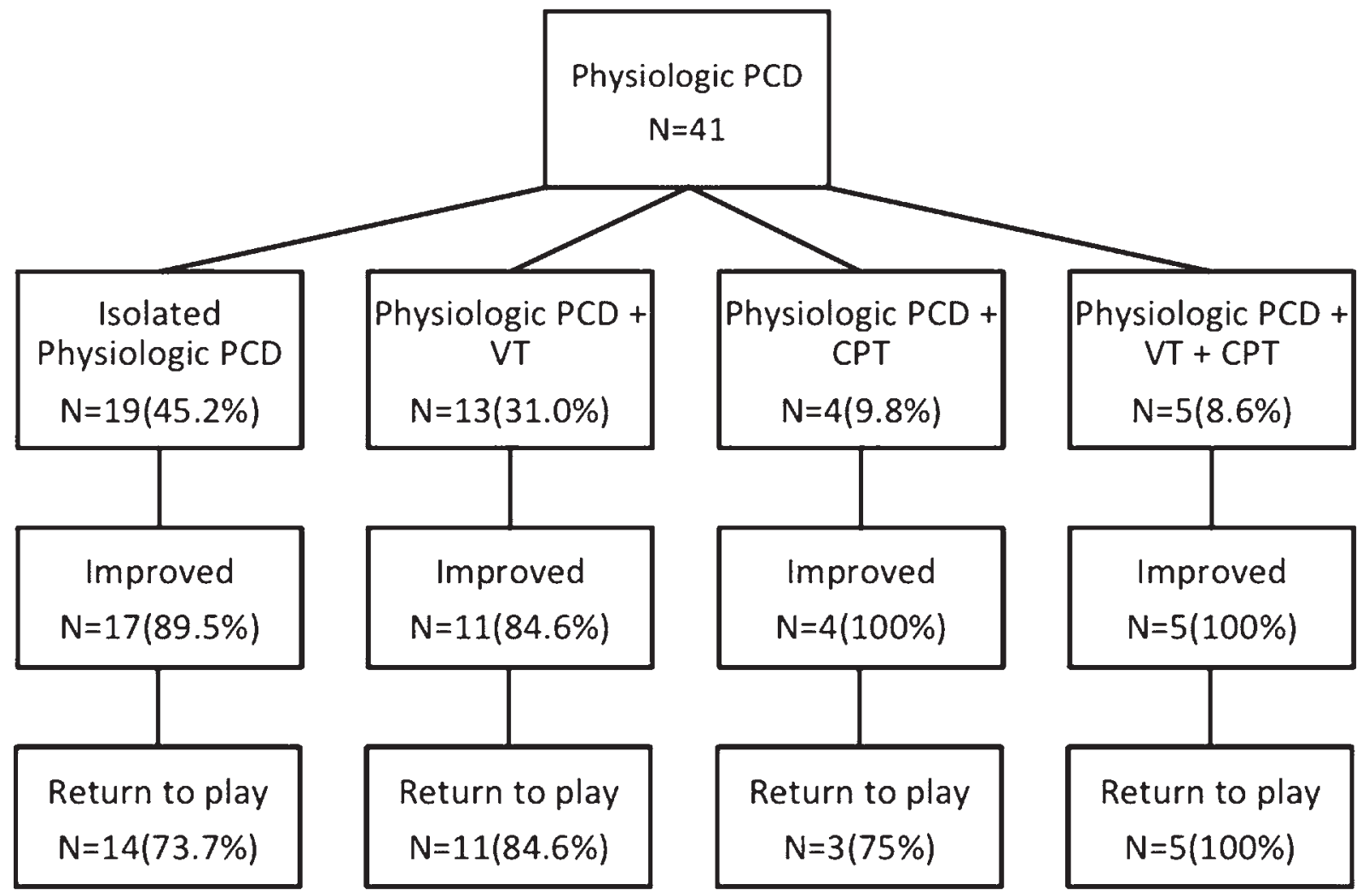

FIG. 2. Clinical outcomes in 41 cases involving pediatric patients with physiological PCD who had complete follow-up and underwent submaximal exercise therapy alone or submaximal exercise therapy and targeted multidisciplinary interventions. CPT = cervical spine physiotherapy; VT = vestibular physiotherapy.

\section{Discussion}

The results of the present study suggest that graded aerobic treadmill testing is safe and well tolerated, and it provides clinically valuable information to aid in the evaluation and management of pediatric SRC and PCS. Furthermore, it suggests that tailored submaximal exercise prescription may be an effective therapeutic intervention for appropriately selected pediatric patients with PCS.

Results of previous studies have suggested that graded aerobic treadmill testing is a safe and well-tolerated clinical tool for use in athletes and nonathletes with PCS as well as concussion patients who have achieved symptomatic recovery. ${ }^{10,23,25,26,28,29}$ Using a modified Balke treadmill protocol, this test exposes patients to incremental increases in aerobic exercise with frequent assessment of concussion symptoms, RPE, and physiological parameters. Because the test is terminated once concussion symptom exacerbation or symptom-free maximal exhaustion is achieved, PCS patients who have not yet achieved full physiological recovery often experience a mild and transient increase in concussion symptoms, ${ }^{23,24,29}$ whereas those who have achieved physiological recovery reach exercise thresholds that are safely achieved during sporting activities..$^{10}$ PCS patients who undergo treadmill testing often demonstrate evidence of exercise intolerance with a mean test duration ranging from 8 to 15 minutes. ${ }^{23,24}$

To date, studies of graded aerobic treadmill testing have been performed largely in adults and older adolescents at a single institution, whereas only limited data are available for children and younger adolescents who make up a significant proportion of SRC patients. In the present study, graded aerobic treadmill testing was found to be safe and well tolerated in a heterogeneous population of pediatric patients with SRC. This study included patients ranging from 11 to 19 years of age who endorsed a wide range and magnitude of clinical symptoms and who underwent testing at variable levels of injury acuteness and severity. There were no complications related to treadmill testing observed in this pediatric cohort. All treadmill tests were completed, with the exception of 3 tests that were terminated early due to transient lower-extremity soreness related to previously known orthopedic injuries. Among SRC patients who were symptomatic and underwent initial treadmill testing for classification of PCS subtype, the mean duration of the first treadmill test was 15.2 minutes (SD 7.1 minutes). Despite symptom provocation, none of the study patients required medical attention for acute or delayed exacerbation of concussion symptoms following treadmill testing. Treadmill testing was also well tolerated by the 2 study patients tested with symptomatic traumatic cranial neuropathies. ${ }^{15,16}$

One of the greatest challenges facing physicians who care for pediatric patients with SRC is deciding when the pathophysiological mechanisms that govern traumatic brain injury have resolved and children and adolescents may safely return to sports. Unfortunately, there are limited clinical tools that can assist physicians with this process. Some authors suggest that neuropsychological testing 
can be used as a supplemental tool to confirm concussion recovery. ${ }^{34}$ However, studies suggest some of the psychometric properties of select computerized neurocognitive testing tools may not meet optimal standards for clinical use. 1,36,37 In contrast, accumulating work suggests that graded aerobic treadmill testing can be used as a clinical tool to confirm physiological recovery following concussion. In 1 study that used a combination of treadmill testing and the Zurich Consensus Conference Guidelines to clear adolescent concussion athletes to resume sports participation, $100 \%$ successfully returned to full sports activities..$^{10}$ Despite the fact that $48 \%$ of patients in that study demonstrated at least 1 borderline or below-average score on computerized neurocognitive testing performed the day of treadmill testing and medical clearance, performance on these tests did not predict successful returnto-play or return-to-learn outcomes. In the present study, 65 pediatric SRC patients underwent graded aerobic treadmill testing to confirm physiological concussion recovery. Of the patients who were deemed fully recovered by treadmill testing and who received medical clearance to return to sports, $100 \%$ made a successful return to full sports activities and none returned to the clinic with recurrent concussion symptoms. Based on the results of treadmill testing, 2 patients were deemed not recovered and were not cleared to return to sports.

In this study, a subgroup of patients underwent graded aerobic treadmill testing and neuropsychological testing to confirm physiological and neurocognitive recovery. Overall, the results of these tests were highly concordant, with some exceptions. Most notable was the finding of 2 patients who were deemed physiologically recovered by treadmill testing but demonstrated significant multidomain cognitive impairments on formal neuropsychological testing. These results suggest that objective impairments in cognitive functioning may exist in some patients who appear physiologically recovered and underscore the need for a multidisciplinary approach to medical clearance.

Emerging research suggests that in addition to being useful as a clinical tool to assess physiological recovery, graded aerobic treadmill testing can be used to classify PCS into operational subtypes or disorders and direct patients to rehabilitation strategies that target the pathophysiological mechanisms governing persistent concussion symptoms. ${ }^{14,28,29}$ Specifically, PCS patients who achieve a symptom-limited threshold on treadmill testing are diagnosed with physiological PCD, a condition initially proposed to be mediated by persistent alterations in autonomic nervous system function and cerebral blood flow. ${ }^{14,27,28}$ Preliminary work has demonstrated alterations in regional resting cerebral blood flow and cerebrovascular responsiveness in adolescent patients with physiological PCD diagnosed by use of brain $\mathrm{MRI} \mathrm{CO}_{2}$ stress testing. ${ }^{35}$ Research also suggests that physiological PCD may be mediated by alterations in $\mathrm{CO}_{2}$ sensitivity and autonomic cerebral blood flow regulation. ${ }^{8}$ In some cases, persistent concussion symptoms that are not exacerbated by treadmill testing can arise from isolated dysfunction of central and peripheral components of the vestibulo-ocular system or the cervical spine. ${ }^{14}$ In other cases, patients with SRC can develop ICHD-3 beta-defined new onset or worsening migraine headaches ${ }^{22,39}$ or postinjury psychiatric outcomes that can take the form of novel psychiatric disorders, novel suicidal ideation, or worsening symptoms of a preinjury psychiatric disorder. ${ }^{17}$ Graded aerobic treadmill testing is especially important because, as a recent study by Leddy et al. suggests, individual concussion symptoms alone are insufficient to allow classification of concussion patients into unique PCDs or trajectories. ${ }^{25}$ In Leddy et al.'s study of primarily adult PCS patients who underwent treadmill testing, approximately $28 \%$ were diagnosed with physiological PCD, while $72 \%$ were diagnosed with vestibuloocular or cervicogenic PCD.

The findings of the present study are in agreement with this previous work and demonstrate that graded aerobic treadmill testing provides clinically valuable information that can be used to classify pediatric PCS patients and direct them to targeted rehabilitation programs. Overall, $96.7 \%$ of initial treadmill tests used to classify PCS subtype provided clinically valuable information, with 2 tests terminated early due to orthopedic injuries yielding indeterminate results. Sixty-one SRC patients underwent graded aerobic treadmill testing for classification of PCS subtype, resulting in the diagnosis of physiological PCD in $95.1 \%$ and the diagnosis of cervicogenic PCD in $1.6 \%$. The distribution of PCDs across this pediatric population differs significantly from the distribution in previous studies involving older patients; this difference may be due to several factors. First, the patients who underwent treadmill testing for PCS classification in this study had more acute injuries than those in the study by Leddy et al. ${ }^{25}$ (mean duration of symptoms 8.4-10.2 months). Second, it is possible that the global metabolic brain injury that characterizes physiological PCD in adults plays a more central role in the pathophysiology of pediatric PCS. This hypothesis is supported by findings that the developing pediatric brain is more sensitive to catastrophic cerebrovascular insults such as second impact syndrome and diffuse cerebral edema $^{33}$ and that younger children may take longer to recover after concussion, ${ }^{31}$ as well as preliminary findings of alterations in resting cerebral blood flow and cerebrovascular responsiveness in adolescent SRC patients who undergo imaging examinations days to months after injury. ${ }^{32,35}$ Taken together, these results suggest that the clinical presentation of pediatric PCS is heterogeneous and that concussion symptoms may be mediated by unique and often overlapping pathophysiological mechanisms. Future research studies employing exercise science and neuroimaging techniques are undoubtedly needed to clarify the relationship between cardiovascular, autonomic, and cerebrovascular alterations that mediate physiological PCD.

At present, there is a paucity of studies examining the role of targeted, multidisciplinary rehabilitative strategies in PCS patients, and there are even fewer studies including pediatric patients..$^{6,738}$ Although some studies have investigated the effect of aerobic exercise programs on symptom scores and return-to-play outcomes following concussion, ${ }^{18,19,30}$ variability in sample size, patient populations, and exercise programs across these studies make it difficult to draw conclusions regarding the effectiveness of this treatment option. In order for future studies to comprehensively evaluate the effect of a targeted therapeutic 


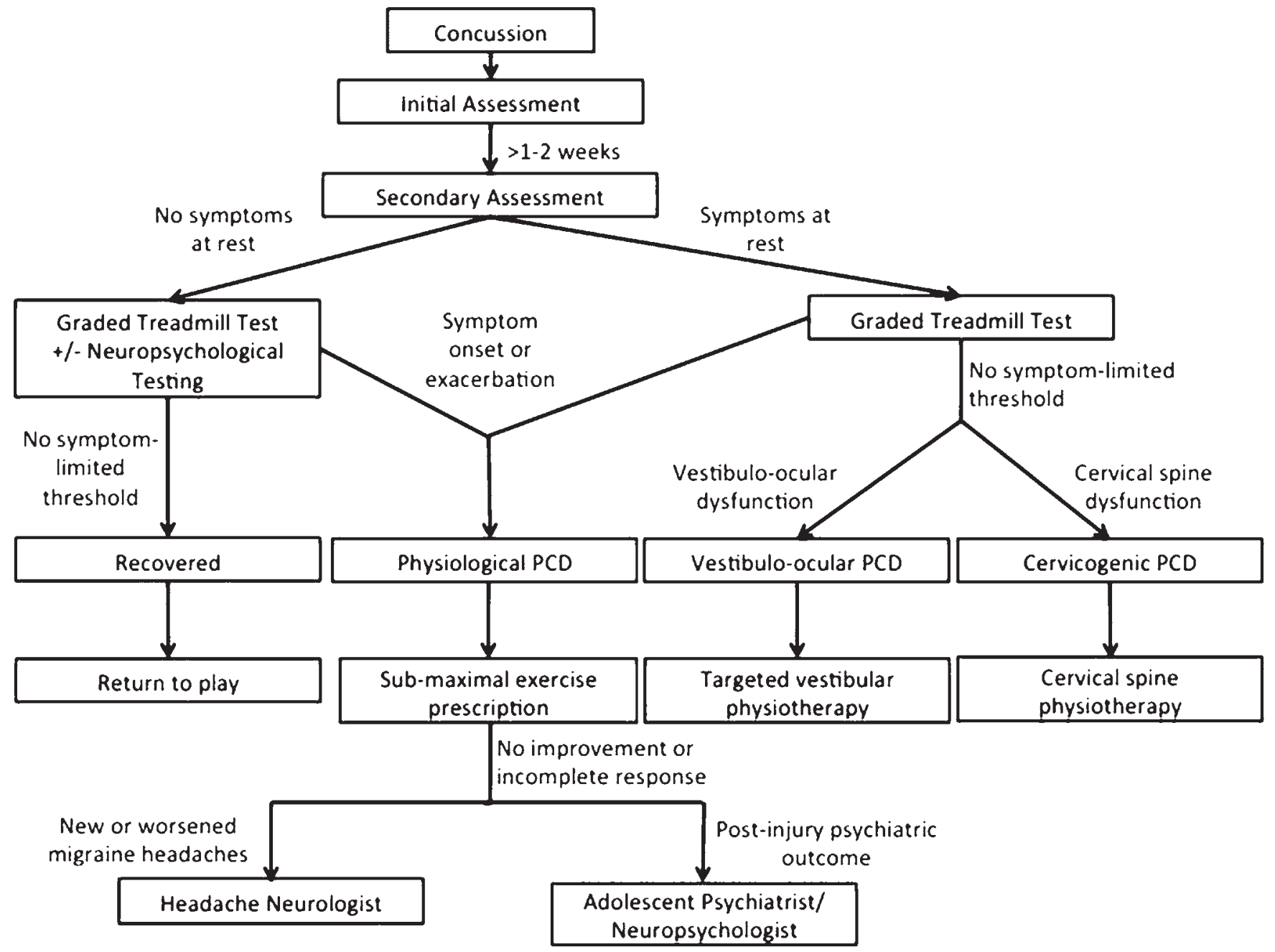

FIG. 3. Summary of institutional multidisciplinary approach to pediatric SRC and PCS. Modified from Ellis MJ, Leddy JJ, Willer B: Physiological, vestibulo-ocular and cervicogenic post-concussion disorders: an evidence-based classification system with directions for treatment. Brain Inj 29:238-248, 2015, with permission from Taylor \& Francis, LLC.

intervention within a heterogeneous PCS population, several key criteria must be met. Firstly, prior to initiation of a given treatment it is important that the study population be appropriately classified and that the pathophysiological mechanisms proposed to underlie persistent concussion symptoms (i.e., exercise intolerance) in these patients be clearly identified using validated diagnostic techniques. Secondly, prior to treatment initiation it is also important to control for other clinical variables that may account for concussion symptoms, that have been found to impact the natural history of concussion recovery, that may be mediated by heterogeneous pathophysiological mechanisms, and that respond differently to treatment (i.e., presence of vestibulo-ocular dysfunction, cervical spine soft tissue injury, migraine headaches, and postinjury psychiatric disorders). Lastly, it is important that studies evaluate the effect of an intervention not only on patient outcomes such as symptom scores and return-to-play status, but also on clinical biomarkers and test results that reflect the underlying pathophysiology the intervention aims to target.

Given these essential requirements, the effect of submaximal aerobic exercise on patient outcomes in this retrospective study is difficult to evaluate. Similar to results observed in adults, 3,26 a significant proportion of patients with physiological PCD and complete follow-up in this study experienced clinical improvement (90.2\%) and complete recovery and successful return-to-play following submaximal exercise treatment (80.5\%). However, conclusions related to the independent effect of this treatment on physiological PCD pathophysiology and patient outcomes are hampered by the fact that treadmill testing was not used to confirm recovery in all patients, compliance with exercise programs was not rigorously assessed, and many patients who met the criteria for physiological PCD also demonstrated evidence of vestibulo-ocular dysfunction and cervical spine soft tissue injury that were treated with other multidisciplinary targeted interventions. Consequently, future prospective controlled studies examining submaximal aerobic exercise prescription in patients with isolated physiological PCD (in those patients with clinically excluded vestibulo-ocular, cervicogenic, migraine, and psychiatric pathology) remain necessary to properly evaluate the independent effect of this treatment strategy on patient outcomes. Future studies should also include the 
use of logbooks or digital watches to determine if patients comply with the frequency and intensity of tailored aerobic exercise programs.

Despite these study limitations, the preliminary clinical experience presented here has had important implications on shaping our evolving institutional approach to the evaluation and management of pediatric patients with acute SRC as well as those with PCS. In our experience, the majority of pediatric patients with SRC will achieve complete neurological recovery and return to school and sports activities within 1-4 weeks with education and conservative management, including proper return-to-learn and returnto-play guidance. Because graded aerobic treadmill testing and formal neuropsychological testing are time-consuming and require access to specialized personnel, we consider these resources on an individual patient basis to assist in return-to-play decision making. Accordingly, in more complex cases involving patients with preinjury conditions such as migraine headaches, ADHD, or mood disorders whose physiological recovery is difficult to assess, those with a significant concussion history (more than 2 physician-diagnosed concussions or more than 1 concussion in the same season), and those who develop PCS, we often consider a combination of graded aerobic treadmill testing and formal neuropsychological testing to confirm full neurological and neurocognitive recovery prior to return-to-play clearance. ${ }^{15}$ Patients who develop PCS are often considered for graded aerobic treadmill testing in order to classify their condition according to PCS subtypes and initiate targeted rehabilitation programs. Although all patients with diagnosed physiological PCD are offered submaximal aerobic exercise programs, the results of this study have led us to consider early pharmacological interventions in patients with physiological PCD who develop ICHD-3 beta-defined new-onset or worsening migraine headaches or postinjury psychiatric outcomes and who appear less responsive to this form of treatment (Fig. 3).

The results of this study must be interpreted in light of several important limitations. Firstly, the study population included pediatric SRC patients referred to a tertiary multidisciplinary pediatric clinic that likely included patients with more severe injuries and who were more likely to develop PCS and other adverse outcomes such as migraine headaches and postinjury psychiatric disorders. Studies performed at tertiary concussion clinics are also more likely to include a significant proportion of patients who are eventually lost to follow-up, which can impact study results. Secondly, although the present study suggests that graded aerobic treadmill testing may help physicians classify and confirm physiological recovery in SRC patients, results of testing were not compared with other objective measures of traumatic brain injury such as formal neuropsychological testing in all patients. Lastly, the retrospective nature of the study, the variability in testing procedures, and the absence of a control group represent other study limitations but highlight important considerations for future research.

\section{Conclusions}

The present study provides preliminary empirical evidence that graded aerobic treadmill testing is a safe, tolerable, and clinically valuable tool to aid in the evaluation and management of pediatric SRC and PCS patients. The results of this study suggest that graded aerobic treadmill testing can provide clinically valuable information that helps confirm physiological recovery in pediatric SRC patients. Furthermore, graded aerobic treadmill testing is helpful in classifying pediatric PCS into operational PCDs that can be targeted with individualized rehabilitation programs. Future prospective studies are warranted to examine whether results of graded aerobic treadmill testing are concordant with other objective neuropsychological and neuroimaging measures of recovery. Research is needed to examine whether the addition of supplemental tools such as graded aerobic treadmill testing, computerized neurocognitive tools, and formal neuropsychological testing offer additional clinical value in comparison with standard graduated return-to-play guidelines. Future prospective studies incorporating exercise science and neuroimaging tools are needed to clarify the pathophysiological mechanisms governing physiological PCD and response to treatment. Finally, the results of this study provide support for a prospective randomized controlled trial of tailored submaximal aerobic exercise prescription in patients with physiological PCD.

\section{Acknowledgments}

All phases of this study were supported by a grant from the Pan Am Clinic Foundation, Winnipeg Jets True North Foundation, and a generous donation by Leonard and $\mathrm{Su}-$ san Asper.

\section{References}

1. Alsalaheen B, Stockdale K, Pechumer D, Broglio SP: Measurement Error in the Immediate Postconcussion Assessment and Cognitive Testing (ImPACT): systematic review. J Head Trauma Rehabil 31:242-251, 2016

2. Babcock L, Byczkowski T, Wade SL, Ho M, Mookerjee S, Bazarian JJ: Predicting postconcussion syndrome after mild traumatic brain injury in children and adolescents who present to the emergency department. JAMA Pediatr 167:156161,2013

3. Baker JG, Freitas MS, Leddy JJ, Kozlowski KF, Willer BS: Return to full functioning after graded exercise assessment and progressive exercise treatment of postconcussion syndrome. Rehabil Res Pract 2012:705309, 2012

4. Boake C, McCauley SR, Levin HS, Contant CF, Song JX, Brown SA, et al: Limited agreement between criteria-based diagnoses of postconcussional syndrome. J Neuropsychiatry Clin Neurosci 16:493-499, 2004

5. Borg G: Perceived exertion as an indicator of somatic stress. Scand J Rehabil Med 2:92-98, 1970

6. Broglio SP, Collins MW, Williams RM, Mucha A, Kontos AP: Current and emerging rehabilitation for concussion: a review of the evidence. Clin Sports Med 34:213-231, 2015

7. Burke MJ, Fralick M, Nejatbakhsh N, Tartaglia MC, Tator $\mathrm{CH}$ : In search of evidence-based treatment for concussion: characteristics of current clinical trials. Brain Inj 29:300305, 2015

8. Clausen M, Pendergast DR, Willer B, Leddy J: Cerebral blood flow during treadmill exercise is a marker of physiological postconcussion syndrome in female athletes. J Head Trauma Rehabil 31:215-224, 2016

9. Corwin DJ, Zonfrillo MR, Master CL, Arbogast KB, Grady $\mathrm{MF}$, Robinson RL, et al: Characteristics of prolonged concus- 
sion recovery in a pediatric subspecialty referral population. J Pediatr 165:1207-1215, 2014

10. Darling SR, Leddy JJ, Baker JG, Williams AJ, Surace A, Miecznikowski JC, et al: Evaluation of the Zurich Guidelines and exercise testing for return to play in adolescents following concussion. Clin J Sport Med 24:128-133, 2014

11. Eisenberg MA, Meehan WP III, Mannix R: Duration and course of post-concussive symptoms. Pediatrics 133:999_ 1006, 2014

12. Ellis MJ, Cordingley D, Vis S, Reimer K, Leiter J, Russell K: Vestibulo-ocular dysfunction in pediatric sports-related concussion. J Neurosurg Pediatr 16:248-255, 2015

13. Ellis MJ, Figley CR, Mutch WA, Massicotte E, Mikulis DJ, Essig M, et al: Neuroimaging in sports-related concussion management: current status and future directions. Curr Res Concussion 1:33-39, 2014

14. Ellis MJ, Leddy JJ, Willer B: Physiological, vestibulo-ocular and cervicogenic post-concussion disorders: an evidencebased classification system with directions for treatment. Brain Inj 29:238-248, 2015

15. Ellis MJ, McDonald PJ, Cordingley D, Mansouri B, Essig M, Ritchie L: Retirement from sport considerations following pediatric sports-related concussion: case illustrations and institutional approach. Neurosurg Focus 40(4):E8, 2016

16. Ellis MJ, Ritchie L, Cordingley D, Essig M, Mansouri B: Traumatic optic neuropathy: a potentially unrecognized diagnosis after sports-related concussion. Curr Sports Med Rep 15:27-32, 2016

17. Ellis MJ, Ritchie LJ, Koltek M, Hosain S, Cordingley D, Chu $\mathrm{S}$, et al: Psychiatric outcomes after pediatric sports-related concussion. J Neurosurg Pediatr 16:709-718, 2015

18. Gagnon I, Galli C, Friedman D, Grilli L, Iverson GL: Active rehabilitation for children who are slow to recover following sport-related concussion. Brain Inj 23:956-964, 2009

19. Gagnon I, Grilli L, Friedman D, Iverson GL: A pilot study of active rehabilitation for adolescents who are slow to recover from sport-related concussion. Scand J Med Sci Sports 26:299-306, 2016

20. Giza CC, Hovda DA: The neurometabolic cascade of concussion. J Athl Train 36:228-235, 2001

21. Grubenhoff JA, Deakyne SJ, Brou L, Bajaj L, Comstock RD, Kirkwood MW: Acute concussion symptom severity and delayed symptom resolution. Pediatrics 134:54-62, 2014

22. Headache Classification Committee of the International Headache Society: The International Classification of Headache Disorders, 3rd edition (beta version). Cephalalgia 33:629-808, 2013

23. Kozlowski KF, Graham J, Leddy JJ, Devinney-Boymel L, Willer BS: Exercise intolerance in individuals with postconcussion syndrome. J Athl Train 48:627-635, 2013

24. Leddy JJ, Baker JG, Kozlowski K, Bisson L, Willer B: Reliability of a graded exercise test for assessing recovery from concussion. Clin J Sport Med 21:89-94, 2011

25. Leddy JJ, Baker JG, Merchant A, Picano J, Gaile D, Matuszak J, et al: Brain or strain? Symptoms alone do not distinguish physiologic concussion from cervical/vestibular injury. Clin J Sport Med 25:237-242, 2015

26. Leddy JJ, Kozlowski K, Donnelly JP, Pendergast DR, Epstein LH, Willer B: A preliminary study of subsymptom threshold exercise training for refractory post-concussion syndrome. Clin J Sport Med 20:21-27, 2010

27. Leddy JJ, Kozlowski K, Fung M, Pendergast DR, Willer B: Regulatory and autoregulatory physiological dysfunction as a primary characteristic of post concussion syndrome: implications for treatment. NeuroRehabilitation 22:199-205, 2007

28. Leddy JJ, Sandhu H, Sodhi V, Baker JG, Willer B: Rehabili- tation of concussion and post-concussion syndrome. Sports Health 4:147-154, 2012

29. Leddy JJ, Willer B: Use of graded exercise testing in concussion and return-to-activity management. Curr Sports Med Rep 12:370-376, 2013

30. Maerlender A, Rieman W, Lichtenstein J, Condiracci C: Programmed physical exertion in recovery from sports-related concussion: a randomized pilot study. Dev Neuropsychol 40:273-278, 2015

31. Makdissi M, Davis G, Jordan B, Patricios J, Purcell L, Putukian M: Revisiting the modifiers: how should the evaluation and management of acute concussions differ in specific groups? Br J Sports Med 47:314-320, 2013

32. Maugans TA, Farley C, Altaye M, Leach J, Cecil KM: Pediatric sports-related concussion produces cerebral blood flow alterations. Pediatrics 129:28-37, 2012

33. McCrory P, Davis G, Makdissi M: Second impact syndrome or cerebral swelling after sporting head injury. Curr Sports Med Rep 11:21-23, 2012

34. McCrory P, Meeuwisse WH, Aubry M, Cantu B, Dvorák J, Echemendia RJ, et al: Consensus statement on concussion in sport: the 4th International Conference on Concussion in Sport held in Zurich, November 2012. Br J Sports Med 47:250-258, 2013

35. Mutch WA, Ellis MJ, Ryner LN, Ruth Graham M, Dufault $\mathrm{B}$, Gregson B, et al: Brain magnetic resonance imaging $\mathrm{CO}_{2}$ stress testing in adolescent postconcussion syndrome. J Neurosurg [epub ahead of print December 18, 2015. DOI: 10.3171/2015.6.JNS15972]

36. Nelson LD, LaRoche AA, Pfaller AY, Lerner EB, Hammeke TA, Randolph C, et al: Prospective, head-to-head study of three computerized neurocognitive assessment tools (CNTs): reliability and validity for the assessment of sport-related concussion. J Int Neuropsychol Soc 22:24-37, 2016

37. Randolph C, McCrea M, Barr WB: Is neuropsychological testing useful in the management of sport-related concussion? J Athl Train 40:139-152, 2005

38. Schneider KJ, Iverson GL, Emery CA, McCrory P, Herring SA, Meeuwisse WH: The effects of rest and treatment following sport-related concussion: a systematic review of the literature. Br J Sports Med 47:304-307, 2013

39. Seifert TD: Sports concussion and associated post-traumatic headache. Headache 53:726-736, 2013

40. Yeates KO, Luria J, Bartkowski H, Rusin J, Martin L, Bigler ED: Postconcussive symptoms in children with mild closed head injuries. J Head Trauma Rehabil 14:337-350, 1999

\section{Disclosures}

The authors report no conflict of interest concerning the materials or methods used in this study or the findings specified in this paper.

\section{Author Contributions}

Conception and design: Ellis, Cordingley, Girardin, Leiter, Russell. Acquisition of data: all authors. Analysis and interpretation of data: all authors. Drafting the article: Ellis, Cordingley, Girardin, Leiter, Russell. Critically revising the article: all authors. Reviewed submitted version of manuscript: all authors. Approved the final version of the manuscript on behalf of all authors: Ellis. Statistical analysis: Russell.

\section{Correspondence}

Michael Ellis, Pan Am Clinic, 75 Poseidon Bay, Winnipeg, MB R3M 3E4, Canada. email: mellis3@panamclinic.com. 